\title{
Análisis de Expresión de Calreticulina en Vena Safena Parva
}

\author{
Calreticulin Expression Analysis in Small Saphenous Vein
}

Sanzana-Cuche, R. ${ }^{1,3}$; Fernández, E. ${ }^{1}$ Leiva, A. P. ${ }^{2}$ \& Rosas, C. C. ${ }^{2,4}$

\begin{abstract}
SANZANA-CUCHE, R.; FERNÁNDEZ, E.; LEIVA, A. P. \& ROSAS, C. C. Análisis de expresión de calreticulina en vena safena parva. Int. J. Morphol., 38(1):182-185, 2020.

RESUMEN: La proteína chaperona Calreticulina (CRT), ha sido identificada en retículo endoplásmico (RE) y últimamente en la matriz extracelular (MEC) de predentina y arterias, atribuyéndole diferentes funciones extracelulares entre las que destacan la adhesión celular, regulación de la MEC y prevención en la formación de trombos. El objetivo del estudio fue identificar la presencia de CRT en MEC de vena safena parva. Se extrajo una muestra de vena safena parva de un espécimen masculino y luego fue procesada por medios histológicos e inmunohistoquímicos para identificar su presencia. Mediante técnicas de inmunohistoquímica se pudo evidenciar la presencia de CRT en la MEC de la adventicia de vena safena parva. La presencia de CRT en MEC de safena parva orienta a que CRT tienen funciones de tipo extracelular en esta localización, pero es necesario realizar estudios más precisos para dilucidar sus principales funciones en la zona.
\end{abstract}

PALABRAS CLAVE: Calreticulina; Vena safena parva; Matriz extracelular.

\section{INTRODUCCIÓN}

La calreticulina (CRT) es una proteína chaperona de $46 \mathrm{kDa}$ que consta de tres dominios estructuralmente y funcionalmente distintos que incluyen sitios de interacción de calcio de alta y baja afinidad, una secuencia de señales para dirigirse al retículo endoplásmico (RE) y una secuencia KDEL para su recuperación / retención en el RE (Obeid et al., 2007; Gold et al., 2010).

Las funciones de CRT al interior del lumen del RE incluyen el plegamiento adecuado de las proteínas y las glicoproteínas, la prevención de la agregación de proteínas, el control de la calidad de las proteínas mediante la identificación y la prohibición de entrada de las proteínas mal plegadas al RE para la destrucción mediada por ubiquitina y la regulación del metabolismo del calcio que influye en la señalización celular (Gold et al.).

Los estudios con ratones deficientes en CRT y células madre embrionarias muestran un papel crítico para la CRT en el mantenimiento del Calcio en el RE, ya que los ratones deficientes en CRT muestran un desarrollo cardíaco anormal y muerte embrionarias. Por otro lado, el aumento de la expresión de la CRT, en ratones transgénicos, produce un aumento significativo de la cantidad de Calcio en el RE, lo cual produce bradicardia, bloqueo cardíaco completo, muerte súbita, edema cardíaco y una estructura anómala del sarcómero del corazón, ventrículos y atrios, paredes ventriculares más delgadas y cardiomiocitos irregulares (Mesaeli et al., 1999; Li et al., 2001).

Se ha demostrado que la CRT se localiza en varios sitios, como el RE, la superficie celular y la matriz extracelular (MEC) (Gold et al.; Toledo et al., 2010). Las funciones en la superficie celular de la CRT incluyen la adhesión celular, el desmontaje de la adhesión focal, la migración celular, el anoikis, la fagocitosis y una posible transducción de señales ecto-CRT y binding-partners a la superficie celular (Gold et al.). La expresión en la superficie celular de CRT también se ha relacionado con la determinación de la respuesta inmune contra el cáncer (Obeid et al.; Aguilar-Guzmán et al., 2014).

La detección de la expresión de CRT en MEC sugiere un papel en la mejora de la formación de MEC. Esta detección se llevó a cabo en un estudio que describe a CRT como un componente de la MEC en la matriz de predentina del diente (Somogyi et al., 2003). Este hallazgo es coherente con la observación reciente de que la proteína CRT está

\footnotetext{
${ }^{1}$ Departamento de Anatomía y Medicina Legal, Facultad de Medicina, Universidad de Chile, Santiago, Chile.

${ }^{2}$ Departamento de Ciencias Morfológicas, Facultad de Medicina y Ciencia, Universidad San Sebastián, Santiago, Chile.

${ }^{3}$ Escuela de Medicina, Facultad de Medicina y Ciencia, Universidad San Sebastián, Santiago, Chile.

${ }^{4}$ Núcleo de Ciencias Biológicas, Facultad de Estudios Interdisciplinarios, Universidad Mayor, Santiago, Chile.
} 
aumentada en los tejidos fibróticos, lo que sugiere que la CRT desempeña un papel en la mejora de la formación de MEC y la resistencia de fibroblastos a anoikis, fuertemente asociada con la fibrogénesis (Frisch \& Francis, 1994). El análisis inmunohistoquímico de la localización de CRT en las arterias de un modelo de aterosclerosis en conejos, sugiere que la CRT se localiza en la MEC después de una lesión / daño durante la remodelación vascular (Gold et al.). La localización de CRT en la MEC se induce además por el tratamiento con ácido ascórbico, que estimula la expresión del colágeno. Dadas las interacciones conocidas de la CRT con colágenos fibrilares, laminina y Trombospondina-1 y su capacidad para modular la actividad de la metaloproteinasa de la matriz, es posible que la CRT en la MEC pueda actuar para modular la estructura o la rotación de la MEC (Gold et al.; Wu et al., 2017). Además, la CRT podría actuar como un enlace molecular entre la MEC y la superficie celular para desencadenar eventos mientras está atada a la MEC. La liberación de CRT de las células lesionadas o moribundas durante las condiciones de estrés e hipoxia y su incorporación a la MEC, podría modificar aún más la estructura de la MEC y la función celular, tanto en la reparación de la herida como en las diversas respuestas a la lesión, incluida la fibrosis (Michel, 2003; Valentijn et al., 2004; Kypreou et al., 2008).

Como la presencia de CRT en la MEC es un nuevo descubrimiento y no está bien descrito, creemos que las funciones asociadas o estimuladas por el CRT en la MEC continuarán desarrollándose. Aunque se ha estudiado la presencia de CRT en la matriz extracelular de los vasos sanguíneos, no se ha investigado en vasos sanguíneos humanos como la vena safena parva (VSP). Es por eso que este trabajo abordará el análisis de la expresión de esta proteína en la VSP.

\section{MATERIAL Y MÉTODO}

Análisis Anatómico. Se extrajo tejido de VSP desde la fosa poplítea derecha de una muestra de un hombre de 45 años de edad. Para acceder a la fosa poplítea, se realizó una disección en hoja de libro. Se trazaron dos líneas horizontales, una línea horizontal de límite inferior se realizó a nivel de la cabeza de la fíbula, mientras que la línea horizontal de límite superior se realizó al nivel de la base de la patela. Las dos líneas horizontales estaban unidas por una línea vertical en sus puntos medios. Se realizó una disección por plano, primero se disecó la epidermis y la dermis juntas exponiendo el tejido celular subcutáneo. Luego, este último se disecó con la fascia superficial incluida para exponer la fascia profunda. Es importante mencionar que la VSP se desplazaba en una envoltura de fascia profunda a este nivel poplíteo, por lo que para extraer la VSP, la fascia profunda también se tuvo que disecar. Se cortó una muestra de $1 \mathrm{~cm}$ de largo de VSP y se almacenó en formalina tamponada neutra al $10 \%$.

Análisis Histológico. Se procedió a fijar muestras de VSP en formalina tamponada al $10 \%$ durante 3 a 5 días y se realizó la técnica de deshidratación, diafanización e inclusión en parafina de las muestras en las que se realizaron cortes histológicos de $5 \mu \mathrm{m}$ de espesor, que se tiñeron con hematoxilina-eosina. Para la inmunohistoquímica, se utilizaron secciones histológicas de VSP de $5 \mu \mathrm{m}$, que se hidrataron para proceder con la recuperación antigénica utilizando Dako Target Retrieval (CA, USA) y luego se bloquearon en una solución de peróxido de hidrógeno en metanol (1:10). Para la detección de Calreticulina, se utilizó un suero inmune policlonal anti-Calreticulina humana en una dilución 1: 1000, que luego se reveló haciendo uso de un anticuerpo secundario conjugado a fosfatasa alcalina y posterior incubación con NBT/BCIP. Para controlar los procesos de inmunomarcaje específicos, se utilizaron anticuerpos anti-CD31 (1: 100) en paralelo. Finalmente, se obtuvieron microfotografías utilizando la cámara Moticam 2500 (Motic, USA).

\section{RESULTADOS}

Se observó a aumento mayor corte transversal de órgano tubular, el cual presentaba 3 túnicas bien definidas, las cuales correspondían a íntima, media y adventicia, respectivamente. La túnica íntima se observó bien definida, con presencia de endotelio y una capa subendotelial fina con escaso tejido conectivo laxo. La túnica media se visualizó con pequeños fascículos de músculo liso, una gran presencia de fibras de tejido conectivo laxo a denso irregular, los cuales se disponían circunferencialmente alrededor del lumen y la túnica íntima. Finalmente, la túnica adventicia se observó bastante desarrollada y altamente vascularizada, presentando vasa vasorum, acompañada de gran cantidad de gruesas fibras colágenas conformando un tejido conectivo denso irregular (Fig. 1).

Mediante inmunohistoquímica anti CRT humana, se pudo comprobar su presencia a nivel intracelular, preferentemente de manera perinuclear, lo que da cuenta de su ubicación canónica en células musculares y células endoteliales de la VSP. Al explorar la matriz extracelular, a nivel de la adventicia, fue posible observar inmunorreacción compatible con la presencia de CRT. A nivel de la matriz extracelular, la ubicación de CRT se centró junto a las fibras colágenas (Fig. 2). 
Mediante inmunohistoquímica anti CD-31, se logró individualizar la presencia de células endoteliales tanto en la túnica íntima de VSM, en contacto directo con el lumen, como también formando la túnica íntima de vasos de pequeño calibre que conforman el endotelio del vasa vasorum (Fig. 3).

Esto indicó que el tejido analizado estaba indemne y adecuado para establecer que la presencia de CRT era propia del sector y no provocada por un proceso de ruptura tisular.

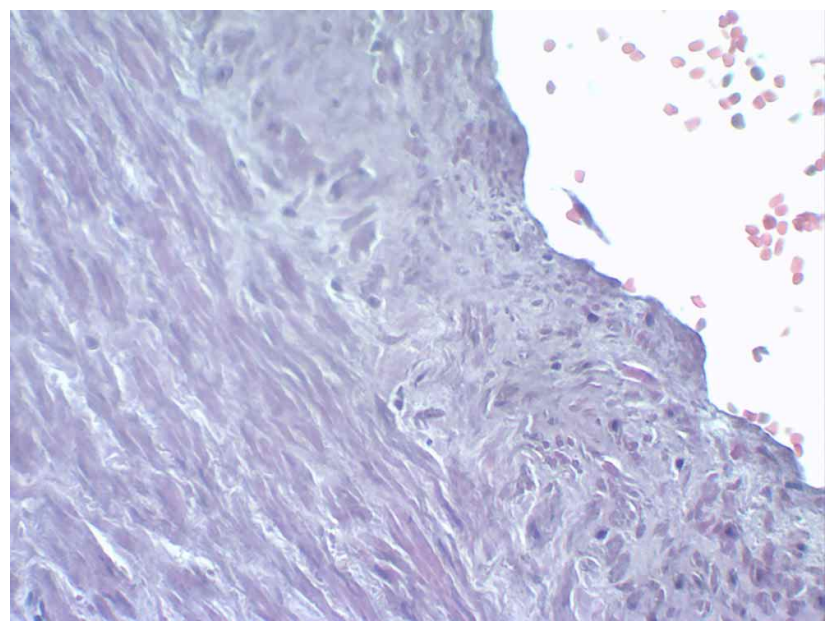

Fig. 1. Corte transversal de vena safena parva teñido con Hematoxilina-Eosina (400x).

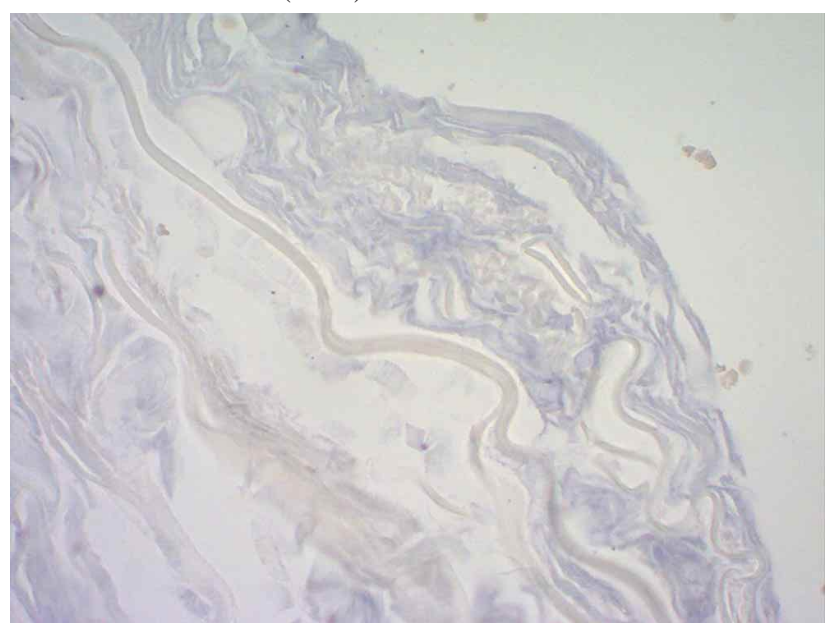

Fig. 2. Expresión de Calreticulina en vena safena parva. Inmunohistoquímica de vena safena parva, la que fue incubada con suero inmune de conejo anti-CRT humana 1:1000 (400x).

\section{DISCUSIÓN}

En el presente estudio, identificamos la presencia de CRT en la matriz extracelular de la adventicia de VSP humana. Esta proteína ha sido habitualmente documentada como una proteína chaperona ubicada dentro del retículo endoplásmico, pero diferentes estudios (Gold et al.; Somogyi

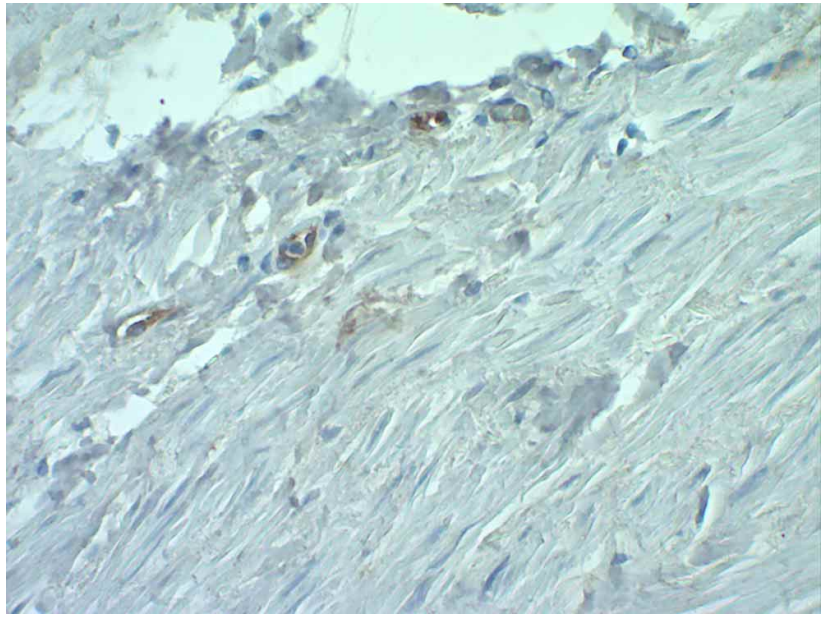

Fig. 3. Expresión de CD-31 en vena safena parva. Inmunohistoquímica de vena safena parva, la que fue incubada con anticuerpos anti-CD31 1:100 (400x).

et al.; Mesaeli et al.) la han ubicado en matriz extracelular dónde podría tener diferentes funciones. Liu et al. (2019) correlacionaron los niveles de CRT con ICAM-1 y VCAM-1 en el suero y líquido sinovial de pacientes con artritis reumatoide, encontrando una elevada co-expresión de CRT con ICAM-1 en las áreas perivasculares del líquido sinovial y una elevada correlación de CRT y VCAM-1 asociada principalmente a la capa de revestimiento sinovial. Además, identificaron, en un modelo de células endoteliales de venas umbilicales humanas, una elevada expresión de ICAM-1 y un aumento en los niveles de fosforilación de Akt y la óxido nítrico sintasa endotelial (eNOS) en presencia de CRT extracelular. Estos hallazgos implican a CRT en procesos asociados a la mantención de una adhesión celular adecuada tanto intercelular como con la matriz extracelular, promoviendo además una adecuada función vascular a través de la correlación con eNOS.

Otra de las funciones que podemos inferir de CRT está asociada a una potente función antiangiogénica de unos de sus dominios N-terminal. En el estudio de Pike et al. (1998) identificaron un dominio antiangiogénico de CRT (CAD180), el cual está constituido por el dominio N-terminal de los aminoácidos 1-180 de CRT, aislado de células de la línea B inmortalizadas de virus de Epstein Barr, el cual sería un potente inhibidor de la angiogénesis. La neovascularización ocular (NO) es una característica frecuente en la retinopatía del prematuro, la retinopatía diabética y la degeneración macular neovascular relacionada con la edad. La NO cursa con una primera fase de pérdida de la microvasculatura retiniana por hipoxia, induciendo un aumento excesivo de factores angiogénicos lo que lleva a una segunda fase de proliferación de vasos aberrantes lo que induce la pérdida de la visión. Por su parte en el estudio de Tu et al. (2018) demostraron las propiedades antiangiogénicas de CAD180 usando un modelo de 
ratones con retinopatía inducida por oxígeno y neovascularización coroidal inducida por láser. Al aplicar CAD180 a los ratones por medio de un virus asociado a adenovirus serotipo 2 que expresara CAD180 a través de vía intra-vítrea, demostraron que CAD180 inhibió significativamente neovascularización retiniana inducida por oxígeno. La expresión de CRT en VSP podría explicarse ayudando en la regulación de procesos angiogénicos. A su vez, Li et al. (2018) identificaron una prevalencia de una mutación de CRT en pacientes con trombosis venosa esplácnica (TVE). Una elevada proporción de pacientes con TVE tienen el diagnóstico de neoplasia mieloproliferativa (NMP), la cual se ha asociado íntimamente a una mutación de la vía JAK2V617F. También se ha identificado que los pacientes con NMP, sin mutación en JAK2V617F son probables candidatos a tener una mutación en CRT (Ianotto et al., 2017) convirtiendo así a CRT en un nuevo blanco diagnóstico de la NMP.

En conclusión, podemos afirmar que CRT está presente en la matriz extracelular de VSP humana y su rol podría estar asociado a la regulación de la adhesión celular y función vascular, mediador antiangiogénico y prevención de formación de trombos. Nuevos estudios que incluyan técnicas de laboratorio más sofisticadas son necesarios para dilucidar cual de todas estas posibles funciones se asocian con la CRT en matriz extracelular de venas.

SANZANA-CUCHE, R.; FERNÁNDEZ, E.; LEIVA, A. P. \& ROSAS, C. C. Calreticulin expression analysis in small saphenous vein. Int. J. Morphol., 38(1):182-185, 2020.

SUMMARY: Calreticulin (CRT) protein, has been identified in the endoplasmic reticulum (ER) and lately in the extracellular matrix (ECM) of predentine and arteries. It is responsible for different extracellular functions, such as cell adhesion, ECM regulation, and the prevention of thrombosis. The aim was to identify the presence of CRT in ECM of small saphenous vein. A sample of small saphenous vein from a male specimen was extracted and then processed by histological and immunohistochemical assays to identify its presence. The presence of CRT in the ECM of the small saphenous vein was observed by immunohistochemical techniques. The presence of CRT in the small saphenous vein ECM, indicates that CRT have extracellular functions in this area, however, more precise studies are necessary to determine its main functions.

KEY WORDS: Calreticulin; Small saphenous vein; Extracellular matrix.

\section{REFERENCIAS BIBLIOGRÁFICAS}

Aguilar-Guzmán, L.; Lobos-González, L.; Rosas, C.; Vallejos, G.; Falcón, C.; Sosoniuk, E.; Coddou, F.; Leyton, L.; Lemus, D.; Quest, A. F.; et al. Human survivin and Trypanosoma cruzi calreticulin act in synergy against a murine melanoma in vivo. PloS One, 9(4):e95457, 2014.
Frisch, S. M. \& Francis, H. Disruption of epithelial cell-matrix interactions induces apoptosis. J. Cell Biol., 124(4):619-26, 1994.

Gold, L. I.; Eggleton, P.; Sweetwyne, M. T.; Van Duyn, L. B.; Greives, M. R.; Naylor, S. M.; Michalak, M. \& Murphy-Ullrich, J. E. Calreticulin: nonendoplasmic reticulum functions in physiology and disease. FASEB J., 24(3):665-83, 2010.

Ianotto, J. C.; Chauveau, A.; Mottier, D.; Ugo, V.; Berthou, C.; Lippert, E. \& Delluc, A. JAK2V617F and calreticulin mutations in recurrent venous thromboembolism: results from the EDITH prospective cohort. Ann. Hematol., 96(3):383-6, 2017.

Kypreou, K. P.; Kavvadas, P.; Karamessinis, P.; Peroulis, M.; Alberti, A.; Sideras, P.; Psarras, S.; Capetanaki, Y.; Politis, P. K. \& Charonis, A. S. Altered expression of calreticulin during the development of fibrosis. Proteomics, 8(12):2407-19, 2008.

Li, M.; De Stefano, V.; Song, T.; Zhou, X.; Guo, Z.; Zhu, J. \& Qi, X. Prevalence of CALR mutations in splanchnic vein thrombosis: A systematic review and meta-analysis. Thromb. Res., 167:96-103, 2018.

Li, Z.; He, L.; Wilson, K. \& Roberts, D. Thrombospondin-1 inhibits TCRmediated T lymphocyte early activation. J. Immunol., 166(4):2427-36, 2001.

Liu, Y.; Wei, W.; Hong, C.; Wang, Y.; Sun, X.; Ma, J. \& Zheng, F. Calreticulin induced endothelial ICAM-1 up-regulation associated with tristetraprolin expression alteration through PI3K/Akt/eNOS/p38 MAPK signaling pathway in rheumatoid arthritis. Mol. Immunol., 107:10-20, 2019.

Mesaeli, N.; Nakamura, K.; Zvaritch, E.; Dickie, P.; Dziak, E.; Krause, K. H.; Opas, M.; MacLennan, D. H. \& Michalak, M. Calreticulin is essential for cardiac development. J. Cell Biol., 144(5):857-68, 1999.

Michel, J. B. Anoikis in the cardiovascular system: known and unknown extracellular mediators. Arterioscler. Thromb. Vasc. Biol., 23(12):2146-54, 2003.

Obeid, M.; Tesniere, A.; Ghiringhelli, F.; Fimia, G. M.; Apetoh, L.; Perfettini, J. L.; Castedo, M.; Mignot, G.; Panaretakis, T.; Casares, N.; et al. Calreticulin exposure dictates the immunogenicity of cancer cell death. Nat. Med., 13(1):54-61, 2007

Pike, S. E.; Yao, L.; Jones, K. D.; Cherney, B.; Appella, E.; Sakaguchi, K.; Nakhasi, H.; Teruya-Feldstein, J.; Wirth, P.; Gupta, G.; et al. Vasostatin, a calreticulin fragment, inhibits angiogenesis and suppresses tumor growth. J. Exp. Med., 188(12):2349-56, 1998.

Somogyi, E.; Petersson, U.; Hultenby, K. \& Wendel, M. Calreticulin--an endoplasmic reticulum protein with calcium-binding activity is also found in the extracellular matrix. Matrix Biol., 22(2):179-91, 2003.

Toledo, V.; Ramírez, G.; Valck, C.; López, N.; Ribeiro, C. H.; Maldonado, I.; Aguilar, L.; Lemus, D. \& Ferreira, A. Comparative in vivo antiangiogenic effects of calreticulin from Trypanosoma cruzi and Homo sapiens sapiens. Biol. Res., 43(3):287-9, 2010.

Tu, L.; Wang, J. H.; Barathi, V. A.; Prea, S. M.; He, Z.; Lee, J. H.; Bender, J.; King, A. E.; Logan, G. J.; Alexander, I. E.; et al. AAV-mediated gene delivery of the calreticulin anti-angiogenic domain inhibits ocular neovascularization. Angiogenesis, 21(1):95-109, 2018.

Valentijn, A. J.; Zouq, N. \& Gilmore, A. P. Anoikis. Biochem. Soc. Trans., 32(Pt. 3):421-5, 2004.

Wu, Y.; Puperi, D. S.; Grande-Allen, K. J. \& West, J. L. Ascorbic acid promotes extracellular matrix deposition while preserving valve interstitial cell quiescence within 3D hydrogel scaffolds. J. Tissue Eng. Regen. Med., 11(7):1963-73, 2017.

\section{Dirección para correspondencia: \\ Dr. Carlos Rosas C. \\ Profesor Asistente \\ Departamento de Ciencias Morfológicas \\ Facultad de Medicina y Ciencia \\ Universidad San Sebastián \\ Santiago - CHILE}

Recibido : 23-07-2019

Aceptado: 13-09-2019

Email: carlos.rosas@uss.cl 